\title{
Relación entre depresión y calidad de vida en pacientes con enfermedades crónicas de un hospital de nivel I de Lima Este
}

\author{
Relationship between depression and quality of life in patients with chronic diseases \\ of a level I hospital in Lima East
}

Missael Gabriel Roque Afray ${ }^{1}$

\begin{abstract}
RESUMEN
La presente investigación tiene como objetivo analizar la relación entre depresión y calidad de vida en pacientes con enfermedades crónicas de un Hospital de Nivel I de Lima Este. El presente estudio es de diseño, no experimental, de corte transversal y de tipo correlacional. Los instrumentos utilizados para la medición fueron: el Cuestionario de Estado de Salud SF36, el cual contiene ocho dimensiones y la Escala de Depresión de Hamilton. La muestra estuvo conformada por 61 pacientes con enfermedades crónicas, de ambos sexos y con edades entre 20 y 80 años. En los resultados se encontró relación inversa altamente significativa entre depresión y las ocho dimensiones de calidad de vida, obteniéndose como función física (rho= -.331; $p<0.01$ ), rol físico (rho = -.407; $p<0.01$ ), dolor corporal (rho= -.448; $p<0.01$ ), salud general (rho= $-.467 ; p<0.01)$, rol emocional (rho= $-.518 ; p<0.01$ ), función social (rho= -.537; $p<0.01)$, vitalidad (rho= $-.637 ; p<0.01)$ y salud mental (rho=. $.566 ; p<0.01)$. Por lo tanto, se concluye que a mayores niveles de depresión, menor será la calidad de vida en los pacientes con enfermedades crónicas de un hospital de Nivel 1 de Lima Este.
\end{abstract}

Palabras clave: Depresión, calidad de vida, enfermedad crónica, adultez

\begin{abstract}
The objective of this research is to analyze the relationship between depression and quality of life in patients with chronic diseases of a Level I Hospital in East Lima. The present study is design, not Experimental, cross-sectional and correlational type. The instruments used for the measurement were: the Health Status Questionnaire SF36, which contains eight dimensions, and the Hamilton Depression Scale. The sample consisted of 61 patients with chronic diseases, of both sexes and with ages between 20 and 80 years. The results showed a highly significant inverse relationship between depression and the eight dimensions of quality of life, obtaining physical function (rho $=-.331 ; p<0.01$ ), physical role (rho $=-.407 ; p<0.01$ ), body pain (rho $=-.448 ; p<0.01$ ), general health (rho $=-.467 ; p<0.01$ ), emotional role (rho $=-.518 ; p<0.01$ ), social function (rho $=-.537) ; p<0.01$ ), vitality (rho $=-.637 ; p<0.01$ ) and mental health ( $r h o=-.566 ; p<0.01$ ). Therefore, it is concluded that the higher the levels of depression, the lower the quality of life in patients with chronic diseases in a Level 1 hospital in Lima East.
\end{abstract}

Keywords: Depression, quality of life, chronic disease, adulthood 


\section{INTRODUCCIÓN}

La depresión es una enfermedad de salud mental que, en la actualidad, se ha convertido en un problema de relevancia social. Según datos de la Organización Mundial de la Salud (OMS, 2017) señaló que aproximadamente, 300 millones de personas, entre el 2005 y el 2015, padecieron de depresión. Esta cifra cada vez está en aumento, pues se estima que en la actualidad 615 millones de personas padecen enfermedades de salud mental, siendo la ansiedad y la depresión las más frecuentes (OMS, 2016).

Por otra parte, Ríos et al. (2008) señalaron que los pacientes con enfermedades crónicas como diabetes, hipertensión, insuficiencia cardíaca, problemas óseos presentan mayores niveles de depresión y, al mismo tiempo, menores niveles de bienestar físico e insatisfacción con la imagen corporal. Del mismo modo, Assalvi, Aparecida y Silva (2010) mencionan que durante el afronte del paciente al diagnóstico médico asignado, suelen presentar índices altos de estrés, burnout, depresión y ansiedad, donde síntomas depresivos priman en más alto grado, asimismo, enfatizan que la calidad de vida es un aspecto importante, ya que es la percepción que tiene un individuo hacia la vida que lleva.

Existen investigaciones nacionales e internacionales que analizaron esta problemática, encontrado los siguientes resultados.

Aggelopouloua et al. (2017) realizaron un estudio para a pacientes con insuficiencia cardíaca hospitalizados en Atenas, Grecia; concluyendo que los pacientes con insuficiencia cardíaca presentan síntomas graves de ansiedad y depresión mala calidad de vida.

Bastidas y Galarza (2016) a través de un estudio buscaron determinar la relación entre los tipos de colostomía según su localización y calidad de vida en los pacientes, condujeron que los tipos de colostomía, según su ubicación y la calidad de vida de los pacientes, se relacionaron significativamente, debido a que estos pacientes experimentan situaciones desagradables por los cambios fisiológicos, dietéticos, psicológicos, estéticos, sociales y económicos que experimentan después de la cirugía.

Villanueva y Casas (2014) en un estudio que buscaban determinar la relación entre depresión y calidad de vida, en un grupo de pacientes con tratamiento de hemodiálisis en un hospital de EsSalud en Lima, hallaron que a mayor depresión, menor es el nivel de calidad de vida en dichos pacientes

En cuanto a depresión, Beck sostiene, con su teoría cognitiva, que los síntomas más destacados de la depresión son los de carácter afectivo y cognitivo, es decir, que los pensamientos negativos rígidos, al mantenerse durante un lapso prolongado de tiempo, forman esquemas los cuales desencadenan en una alteración mental, dejando a la persona vulnerable a un trastorno depresivo. Cabe mencionar que esta no presenta ninguna crisis, hasta la aparición de un evento o situación estresante (citado por Butcher, Mineka y Hooley, 2006).

Por otro lado, el modelo teórico biopsicosocial, propuesto por Oblitas (2016), señala que la salud y patología son factores multidisciplinarios y multicausales en base al contexto social, ambientefísico, circunstancias socioeconómicas, factores climáticos, y otros más. De esta manera, la salud sería un estado y un proceso que se alcanza cuando hay armonía biológica, psicológica y con el entorno sociambiental. Del mismo modo, Tuesta (2012) propuso que Calidad de vida está relacionada con la salud y es la mirada de bienestar del paciente hacia sí mismo, también es un factor muy considerado en el tratamiento, como parte de la recuperación y es constituido por 8 dimensiones que son:

Función física: nivel que la carencia de salud restringe el adecuado desempeño de las actividades físicas y las actividades correspondientes a la vida cotidiana; estas tienen relación con el autocuidado, como: caminar, subir escaleras, inclinarse, coger - llevar cargas y la realización de esfuerzos moderados o fuertes.

Rol físico: hace referencia al nivel en que la falta de salud dificulta las actividades laborales y hogareñas, causando disminución o hasta limitación.

Dolor corporal: es el grado del dolor padecido, por otra parte, la medición refiere evaluar los efectos las actividades tanto en el hogar como en las labores ocupacionales, al incrementar los niveles de dolor corporal afectaría a la satisfacción total de la persona. 
Salud general: es el grado en que se percibe su vida referente a la salud y las expectativas hacia las enfermedades, por otro lado, la presente dimensión estará ligada a las demás dimensiones, generando un bienestar físico, mental y social.

Rol emocional: el nivel de afección que deja alteraciones emocionales y las limitaciones que contraen las mismas en el área laboral y otras actividades cotidianas.

Función social: hace referencia al grado que las áreas físicas, emocionales y conductuales afectan y deterioran la interacción con el medio, generando inhibición, tristeza y falta de interés por relacionarse.

Vitalidad: se refiere a la energía que tiene la persona después de continuas actividades, el panorama que se tiene de la vida.

Salud mental: que hace referencia a la sintomatología como la depresión, la ansiedad, el control de la conducta y el control emocional, debido a que todo ello va relacionado con la promoción del bienestar en general.

En cuanto al objetivo, la presente investigación busca determinar la relación significativa entre depresión y calidad de vida en pacientes con enfermedades crónicas de un Hospital de Nivel I de Lima Este.

\section{MATERIALES Y MÉTODOS}

\section{Diseño:}

El diseño de la investigación fue de diseño no experimental, porque no se manipuló la variable de estudio. Además, es de corte transversal porque la recopilación de datos se realizó en un tiempo determinado. Asimismo, es de alcance correlacional, ya que se buscó explorar la relación entre las variables de estudio (Hernández, Fernández y Baptista, 2006).

\section{Participantes:}

La muestra escogida provino de un hospital nivel 1 de Lima Este, durante los meses de noviembre y diciembre. Colocar el tipo de selección. Finalmente, la muestra estuvo conformada por 61 pacientes de consulta externa, entre los 20 y 80 años de edad, de ambos sexos, que padezcan una o más enfermedades crónicas, seleccionado mediante un muestreo no probabilístico intencional.

Con relación a los datos sociodemográficos de los pacientes evaluados, se observa que el $67.2 \%$ de participantes fueron mujeres. En cuanto a la edad, el $45.9 \%$ tiene entre $41 \mathrm{y}$ 65 años. Por otra parte, en el estado civil, el $65.6 \%$ es casados o conviviente y, en el nivel de estudios, un $32.8 \%$ de pacientes denotan haber culminado la secundaria completa. Respecto a enfermedades crónicas, un $54.1 \%$ son personas que presentaron una sola enfermedad crónica.

\section{Instrumentos:}

En cuanto a los instrumentos, se utilizó el Cuestionario de Salud SF 36 para medir los niveles de calidad de vida y la Escala de Depresión Hamilton (HDS) para la variable depresión. El Cuestionario de Salud SF 36 fue diseñado por el Health Institute New England Medical center de Boston en Massachusett. Esta escala consta de 36 preguntas que pretende medir 8 conceptos genéricos sobre la salud: función física (5 Ítems), rol físico (4 Ítems), función social (2 Ítems), rol emocional (3 Ítems), dolor corporal (2 Ítems), salud general (5 Ítems), salud mental (5 Ítems) y vitalidad (4 Ítems).

En cuanto a la calificación, puede darse con la obtención del puntaje total o parcial, asimismo la puntuación abarca desde 0 , como peor estado de salud; hasta 100, mejor estado de salud. Los resultados obtenidos serán debidamente ubicados en un rango que considera lo siguiente: 0-20: deficiente; 21-40: malo; 41-60: regular; 6180: bueno; 81-100: excelente.

Este cuestionario fue validado por Alfaro (2011) Lima en una muestra de 100 pacientes con enfermedad renal crónica en cuatro centros de diálisis de la capital, hallando una confiabilidad, según alfa de Cronbach, de 0.70 y en cuanto a la validez del instrumento, todas las correlaciones ítem-test fueron superiores a .40 .

Para este estudio, se sacó la validez y confiabilidad del instrumento, llegando a obtener en la muestra estudiada un valor de alfa de cronbach de .930, el cual puede ser valorado como indicador de una elevada fiabilidad; Asimismo, los coeficientes, que resultan de la correlación entre las dimensiones, son significativos en su mayoría, lo cual evidencia que el inventario presenta validez de constructo. 
Respecto a la Escala de Depresión Hamilton (HDS), fue elaborada por Hamilton en 1960, siendo un instrumento que constaba de 24 ítems. En 1967 se hizo una revisión por el mismo autor y se reduce a la cantidad de 21 ítems, cabe resaltar que en la actualidad se mantiene una adaptación española que redujo la escala a 17 ítems. El instrumento ofrece datos cuantitativos referentes al trastorno depresivo y su intensidad, permite verificar si el plan de intervención o tratamiento está dando resultado. Las dimensiones identificadas serían: el índice de melancolía, índice de ansiedad, índice de alteraciones del sueño y el índice de somatización. En cuanto a la fiabilidad, posee una buena consistencia interna con un alfa de Cronbach de 0.92 (Ramos-Brieva y Cordero, 1986).

La corrección de cada reactivo se puntúa de 0 a 2 puntos en unos ítems y de 0 a 4 en otros, dando como resultado un puntaje global que será ubicado en el rango: 0-6: sin depresión; 7-17: depresión ligera; 18-24: depresión moderada; 25-52: depresión grave (RamosBrieva y Cordero, 1986).

Para el presente estudio se sacó la validez y confiabilidad del instrumento, llegando a obtener un coeficiente alpha de Cronbach de .80, el cual puede ser valorado como indicador de una elevada fiabilidad. Asimismo, los reactivos que resultan de la correlación entre la única dimensión del instrumento, son moderados y significativos en su totalidad, lo cual evidencia que el inventario presenta validez de constructo.

\section{RESULTADOS}

En la tabla 1 se puede apreciar que el $37.7 \%$ de los pacientes, con enfermedades crónicas, presentaron un nivel excelente en la dimensión función física, lo que significa que los pacientes pueden realizar actividades diarias como el cuidado personal, transportar cargas livianas y realizar esfuerzos moderados necesarios en sus actividades cotidianas. Sin embargo, en la dimensión rol físico, el $42.6 \%$ de los pacientes presentan un nivel deficiente, es decir, tienen dificultades para realizar actividades laborales que requieren mayor esfuerzo físico. Respecto a la dimensión dolor corporal, el $42.6 \%$ de los pacientes presentaron un nivel regular, es decir, el dolor corporal producto de la enfermedad que padece dificulta medianamente sus actividades laborales y domésticas. Del mismo modo, en la dimensión salud general, el $32.8 \%$ de los pacientes se ubicaron en un nivel regular, lo que implica una autovaloración personal que varía según el estado de salud, guardando esto relación con la falta de bienestar para el afronte de problemas venideros, correspondientes a su salud. Por otro lado, se apreció que hay un grupo de pacientes $(27.9 \%)$ se encuentra en un nivel malo de vitalidad, esto quiere decir que los pacientes se sienten cansados y desanimados. Asimismo, en la dimensión función emocional, el $34.4 \%$ de los pacientes se ubicaron en el nivel deficiente, evidenciando dificultades para culminar sus actividades laborales y cotidianas que antes desempeñaban con más esmero, tiempo y cuidado. Respecto a la función social, el $29.5 \%$ de los pacientes se ubicaron en un nivel bueno, esto indica que los pacientes están llevando su vida social habitual con normalidad. Por último, en la dimensión salud mental, el $44.3 \%$ de los pacientes se ubicaron en la categoría regular, es decir, los pacientes presentan ligeros indicadores de tristeza, pérdida de interés, poca energía, ansiedad y preocupaciones.

Tabla 1

Niveles de calidad de vida de los pacientes de un Hospital Nivel 1 de Lima Este, 2017.

\begin{tabular}{llllllllllll} 
Deficiente & & \multicolumn{3}{c}{ Malo } & \multicolumn{4}{c}{ Regular } & \multicolumn{2}{c}{ Bueno } & \multicolumn{2}{c}{ Excelente } \\
& $\mathrm{n}$ & $\%$ & $\mathrm{n}$ & $\%$ & $\mathrm{n}$ & $\%$ & $\mathrm{n}$ & $\%$ & $\mathrm{n}$ & $\%$ \\
\hline Función física & 4 & $6.6 \%$ & 10 & $16.4 \%$ & 15 & $24.6 \%$ & 9 & $14.8 \%$ & 23 & $37.7 \%$ \\
Rol físico & 26 & $42.6 \%$ & 6 & $9.8 \%$ & 7 & $11.5 \%$ & 8 & $13.1 \%$ & 14 & $23 \%$ \\
Dolor corporal & 5 & $8.2 \%$ & 19 & $31.1 \%$ & 26 & $42.6 \%$ & 7 & $11.5 \%$ & 4 & $6.6 \%$ \\
Salud general & 12 & $19.7 \%$ & 19 & $31.1 \%$ & 20 & $32.8 \%$ & 10 & $16.4 \%$ & 0 & $0 \%$ \\
Vitalidad & 4 & $6.6 \%$ & 17 & $27.9 \%$ & 23 & $37.7 \%$ & 12 & $19.7 \%$ & 5 & $8.2 \%$ \\
Función social & 3 & $4.9 \%$ & 11 & $18 \%$ & 12 & $19.7 \%$ & 18 & $29.5 \%$ & 17 & $27.9 \%$ \\
Función emocional & 21 & $34.4 \%$ & 11 & $18 \%$ & 0 & $0 \%$ & 17 & $27.9 \%$ & 12 & $19.7 \%$ \\
Salud mental & 2 & $3.3 \%$ & 13 & $21.3 \%$ & 27 & $44.3 \%$ & 14 & $23 \%$ & 5 & $8.2 \%$ \\
\hline
\end{tabular}


Relación entre depresión y calidad de vida en pacientes con enfermedades crónicas de un hospital de nivel I de Lima Este

En la tabla 2 se observa que el $59 \%$ de los pacientes evidencian depresión ligera que implica cierta apatía, falta de energía e interés, pero no afecta de forma significativa sus actividades cotidianas.

Tabla 2

Niveles de depresión en pacientes de un Hospital Nivel 1 de Lima Este

\begin{tabular}{lll} 
Niveles & $\mathrm{n}$ & $\%$ \\
\hline Sin depresión & 15 & $24.6 \%$ \\
Depresión ligera & 36 & $59 \%$ \\
Depresión moderada & 7 & $11.5 \%$ \\
Depresión grave & 3 & $4.9 \%$ \\
\hline
\end{tabular}

Con el objetivo de analizar y contrastar las hipótesis planteadas, se realizó la prueba de normalidad de ajuste de Kolmogorov-Smirnov (K-S). Como se observa en la tabla 3, los datos correspondientes a las 2 variables no presentan una distribución normal, dado que el coeficiente obtenido (KS) es significativo $(p<0.05)$; por lo tanto, para los análisis estadísticos se empleará la prueba estadística no paramétrica rho de Spearman.

Tabla 3

Prueba de K-S de bondad de ajuste a la curva normal para las variables de estudio

\begin{tabular}{llllll} 
Variable & Dimensiones & Media & D.E & K-S & p \\
Depresión & Depresión global & 12.25 & 7.252 & 0.106 & .088 \\
& Función social & 6.95 & 2.004 & 0.146 & .002 \\
& Función física & 22.97 & 5.651 & .147 & .002 \\
& Rol físico & 5.64 & 1.664 & 0.264 & .000 \\
& Rol emocional & 4.33 & 1.151 & 0.220 & .000 \\
& Dolor corporal & 6.02 & 2.021 & 0.175 & .000 \\
& Salud general & 13.41 & 3.947 & 0.099 & .200 \\
& Salud mental & 15.72 & 3.661 & 0.088 & .200 \\
& Vitalidad & 11.89 & 3.322 & 0.142 & .004 \\
\hline
\end{tabular}

Como se puede apreciar en la tabla 4, el coeficiente de correlación de rho de Spearman indica que existe relación negativa altamente significativa entre la depresión y las dimensiones de calidad de vida, donde los coeficientes de rho oscilan entre -..331; -.637; $p<0.01$. Esto quiere decir que, cuanto mayor es la depresión, menor será la calidad de vida de los pacientes.

Tabla 4

Coeficiente de correlación entre depresión y calidad de vida

\begin{tabular}{lll} 
Calidad de vida & \multicolumn{2}{c}{ Depresión } \\
\cline { 2 - 3 } & Rho & p \\
Función física & -.331 & .001 \\
Función social & -.537 & .000 \\
Rol físico & -.407 & .001 \\
Rol emocional & -.518 & .000 \\
Dolor corporal & -.448 & .000 \\
Salud general & -.467 & .000 \\
Salud mental & -.566 & .000 \\
Vitalidad & -.637 & .000 \\
\hline
\end{tabular}




\section{DISCUSIÓN}

Los resultados que se obtienen, en esta investigación, evidencian que existe relación significativa inversa entre las variables depresión y la calidad de vida en los pacientes con enfermedades crónicas del Hospital Nivel 1 de Lima Este. Es decir que, mientras mayores fueron los niveles de depresión, menor fue la calidad de vida, así como las dimensiones que la componen. Este resultado se asemeja al encontrado por Villanueva y Casas (2014) quienes evidenciaron correlación significativa inversa entre depresión y calidad de vida en un grupo de pacientes con tratamiento de hemodiálisis. Por otra parte, Meyer et al. (2005) señalaron que el cansancio muscular, la actividad física o esfuerzos desmedidos dan origen al cansancio excesivo y problemas de sueño, no por padecer problemas emocionales, sino por sintomatología y cuidado de la propia enfermedad.

En cuanto a la dimensión función física, se encontró relación negativa altamente significativa con la depresión (rho= $-.331 ; \mathrm{p}<0.01$ ), por lo que se puede decir que, a mayores niveles de depresión, menor será la función física de los pacientes. Por ello, al disminuir los niveles de depresión, se podrá observar mejor función física, siendo favorable para realizar actividades cotidianas que no requieren esfuerzo excesivo como subir escaleras, coger objetos, trasladarse de un lugar a otro. En este sentido, Strohle (2009) mencionó que los individuos depresivos y ansiosos experimentan falta de control y sensaciones de malestar en sus vidas cotidianas y perciben que no cuentan con las competencias suficientes para realizar actividades de las que solían hacer con frecuencia y, mucho menos, para realizar actividades extras.

Asimismo, la dimensión rol físico se relacionó de manera negativa altamente significativa con la depresión (rho= -.407; $p<0.01$ ), es decir, al incrementarse los niveles de depresión, se acrecentaban los niveles de calidad en el rol físico de los pacientes. Por ello, a niveles altos de depresión, menores niveles de esfuerzo elevado en actividades laborales y menor producción, eficiencia y rendimiento en las mismas. Mingote, Gálvez, Del Pino y Gutiérrez (2009) manifestaron que los síntomas emocionales tales como ánimo irritable, desinterés, sensibilidad a la crítica de compañeros, tendencia al aislamiento, pérdida de la capacidad de disfrute y frecuentes sentimientos de vacío crónico son, además de conflictos propios del área emocional, potentes limitantes en los roles y las responsabilidades que requieren esfuerzo físico para realizar actividades y labores pesadas.

En cuanto a la dimensión dolor corporal se halló que, a mayor nivel de depresión en el paciente, menor será el dolor físico que experimentará (rho= -.448; p<0.01). Es decir, un estado depresivo grave en el paciente estará relacionado con el desinterés e insensibilidad al dolor corporal padecido, ya que la depresión grave altera las áreas sensitivas de la persona. Apoyando a lo mencionado, Von Korff y Simón (1996) afirmaron que el estado depresivo no parece generar dolor físico en la persona y menos cuando se hace referencia al tiempo que esta persona padece dicho dolor. Asimismo, Beck et al. (2002) señalaron que los pacientes depresivos experimentan un dolor psíquico no necesariamente corporal. En otras palabras, la capacidad para tolerar el dolor padecido por la enfermedad pasa a un segundo plano, toma prioridad en el paciente su estado de ánimo depresivo.

Con respecto a la dimensión salud general y depresión, se evidencia una relación altamente significativa inversa (rho= -.467; $p<0.01$ ), de esta manera, cuando los niveles de depresión se encontraron elevados, se evidenció menor grado de salud general. Como parte de la relación entre ambas variables, se presentan poca valoración de la salud, así como las perspectivas al futuro y la resistencia a enfermarse, por otro lado, al tener relación con otras áreas como bienestar físico, mental y social, la presente área se podría ver disminuida por cualquier factor que altera el bienestar de la persona. Apoyando a lo mencionado, Lugo, Reynoso y Fernández (2014) mencionaron la relación entre la percepción y la valoración del impacto negativo en la vida del paciente con asma crónico está asociado a sintomatología depresiva y ansiosa, y que las repercusiones emocionales de la enfermedad tienen influencia en el apego o abandono que tendrá él mismo al tratamiento.

También se halló relación negativa y altamente significativa entre dimensión vitalidad y la depresión (rho= $-.637 ; p<0.01$ ), de manera que, a mayores niveles de depresión, menores los niveles de calidad en la vitalidad de los pacientes. Se infiere que la magnitud de energía, fuerza y vitalidad que experimentarían los pacientes se vería disminuida con niveles de depresión altos, 
evidenciando: cansancio, alteraciones de sueño, pérdida de interés y demás. Asimismo, un factor determinante que mantiene la depresión en niveles altos y la vitalidad en niveles menores es la calidad de sueño, ya que en esta se refleja la recuperación de energía y fuerza del paciente ante la actividad realizada en el día. De acuerdo a lo expuesto, Mera (2007) califica a la vitalidad como la energía que experimentan los pacientes tras realizar sus actividades, esto se pudo apreciar después de una sesión en pacientes con hemodiálisis, donde se observó que los niveles de intensidad de la terapia genera bienestar en los pacientes, y con ello una mejor percepción y energía para confrontar los tratamientos venideros

En cuanto, a la dimensión función social, se encontró relación negativa altamente significativa con la depresión ( $r h o=-.537 ; p<0.01$ ) presentando que, mientras mayor es la depresión, menor es la función social en los pacientes. Es decir, que los niveles altos de depresión guardarían relación con la inadecuada relación interpersonal que el individuo mantiene en su vida habitual, siendo esto influenciado por dolencias físicas o carencias afectivas-emocionales. Asimismo, Barlés (2000) mencionó que mientras la depresión se inicia en la persona, el área social no genera tantos cambios evidentes, por otro lado, los cambios notables en esta dimensión, se harán más observables, cuando la depresión se encuentre en un nivel moderado o alto, asimismo también tendrán afección en las áreas laborales y sociales.

Del mismo modo, la dimensión de rol emocional se relacionó de manera negativa y altamente significativa con la depresión ( $\mathrm{rho}=-.518$; $p<0.01$ ), es decir, a mayor depresión, menor rol emocional en los pacientes crónicos, observándose que al incrementarse los niveles depresión en el paciente crónico, se da un menor cuidado y la reducción de tiempo a las actividades cotidianas, debido a dificultades emocionales, solo serían parte de la relación entre ambas variables. Piqueras, Ramos, Martínez y Oblitas (2009) resaltan la influencia de las emociones negativas en la salud mental y física, y que las emociones negativas en un nivel alto tales como la tristeza-depresión, miedo-ansiedad, ira y asco que son factores contribuyentes en el proceso de salud-enfermedad. Asimismo señalaron que producto de ello surgen limitaciones durante las actividades laborales, ello debido a la pérdida de interés y placer en las mismas.
Por último, se encontró que, a mayor depresión, menor salud mental en los pacientes crónicos. $(r h o=-.566 ; p<0.01)$. Así, los resultados dejan ver que un nivel alto de depresión guardaría relación con indicadores como la sensación de nerviosismo, bajo estado de moral, desinterés e infelicidad; por otro lado, una adecuada salud mental estaría relacionada a la prevención de trastornos mentales futuros. Apoyando a los resultados encontrados, Rudnicki (2006) sustenta que las principales características emocionales están asociadas a la forma cómo los pacientes, con insuficiencia renal crónica, se enfrentan al tratamiento de hemodiálisis, asimismo, mencionó que los pacientes con la presente enfermedad muestran diferentes sintomatologías pasajeras como: fatiga, apatía, inquietud, irritabilidad y disturbios de atención. Por otro lado, de manera frecuente, presentan depresión, frustración y síntomas de ansiedad relacionado a la salud física, deseo de abandono y encontrar otra forma de tratar la enfermedad.

La presente investigación, con los datos y resultados sobre la depresión y la calidad de vida en pacientes con enfermedades crónicas, llega a la conclusión que existe relación significativa e inversa entre la depresión y calidad de vida en los pacientes con enfermedades crónicas, siendo que, mientras mayor depresión se muestra, menor será el nivel de calidad de vida y sus dimensiones.

\section{Declaración de financiamiento y de conflictos de interés:}

El estudio fue financiado por los autores quienes declaran no tener conflictos de interés.

\section{Correspondencia}

Missael Gabriel Roque Afray.

Dirección: Institución Educativa Particular, Ilo, Moquegua, Perú

Correo electrónico: missael.ra@upeu.edu.pe 


\section{REFERENCIA}

Aggelopouloua A., Nikolaos V., Chatziefstratioua A., Giakoumidakisb K., Elefsiniotisb I., \& Brokalakia H. (2017). El nivel de ansiedad, depresión y calidad de vida en los pacientes con insuficiencia cardíaca en Grecia. Applied Nursing Research, 34(1), 52-56. Recuperado de http://www.sciencedirect.com/science/article/pii/ S0897189716301367

Alfaro, P. (2011). Calidad de vida relacionada a la salud y locus de control de la salud en un grupo de pacientes con tratamiento de hemodiálisis (Tesis de Licenciatura) Pontificia Universidad Católica del Perú, Lima. Recuperado de: http://tesis.pucp.edu.pe/ repositorio/bitstream/handle/123456789/1287/ ALFAR O_CARDENAS_PEDRO_CALIDAD. pdf?sequence $=1$

Assalvi, K., Aparecida, D., y Silva. A. (2010). Evaluación de la calidad de vida y de la depresión de técnicos y auxiliares de enfermería. Revista LatinoAmericana de Enfermagem, 18(3), 126 133. Recuperado de: http://www.scielo.br/ pdf/rlae/v18n3/es_17.pdf

Barlés, C. (2000). Depresión. Medicina Naturista, 1(1), 36-41. Recuperado: https://dialnet.unirioja. es/descarga/articulo/202436.pdf

Bastidas, J, y Galarza, J. (2016) Tipos de colostomía según su localización y la calidad de vida en los pacientes colostomizados que asisten al Consultorio Externo de Cirugía General del Hospital Nacional Guillermo Almenara Irigoyen, Lima, 2015. (Tesis para optar el título profesional en Enfermería). Universidad Peruana Unión. Lima.

Beck, A., Rush, J., Shaw, B., y Emery, G (2002). Terapia Conductual Racional Emotiva. España: Desclee de Brouwer.

Butcher, J., Mineka, S., y Hooley, J. (2006). Psicología Clínica. España: Pearson Educación SA.

Hernández, R., Fernández, C., y Baptista, M. (2006). Metodología, de la investigación. México. El comercio

Lugo, I., Reynoso, L., y Fernández, M. (2014) Percepción de enfermedad, depresión, ansiedad y control del asma: Una primera aproximación. Neumología y cirugía de tórax, 73(2), 114-121. Recuperado: http:// www.scielo.org. $\mathrm{mx} / \mathrm{scielo}$.php? script $=\mathrm{sci}$ arttext\&pid=S0028-37462014000200003

Mera, M. (2007). Calidad de vida de pacientes con Insuficiencia renal crónica terminal en tratamiento sustitutivo con hemodiálisis (Tesis inédita de Licenciatura) Universidad
Austral de Chile. Recuperado de http://cybertesis. uach.cl/tesis/uach/2007/fmm552c/doc/fmm552c. pdf

Meyer, F., Lossnitzer, D., Kristen, A., Schoene, A., Kübler, W., Katus, H., y Borst, M. (2005). Respiratory muscle dysfunction in idiopathic pulmonary arterial hypertension. The European respiratory journal. 25(1), 125-130. Recuperado de: https://www.ncbi.nlm.nih.gov/ pubmed/15640333\#

Mingote, J., Gálvez, M., Del Pino, P., y Gutiérrez, D. (2009). El paciente que padece un trastorno depresivo en el trabajo. Medicina y Seguridad del Trabajo, 55(214), 41-63. Recuperado de: http://scielo.isciii.es/scielo.php?script=sci arttext\&pid=S0465- 546X2009000100004

Oblitas, L. (2006). Psicología de la Salud y calidad de vida. México: Thomson Organización Mundial de Salud (13 abril de 2016). La inversión en el tratamiento de la depresión y la ansiedad tiene un rendimiento del $400 \%$. Washington: Banco Mundial. Recuperado de: http://www.who.int/ mediacentre/news/releases/2016/depressionanxiety- treatment/es/

Organización Mundial de Salud (13 de marzo de 2017). La depresión encabeza la lista de las causas de enfermedad. Washington, EEUU: OMS/Banco Mundial. Recuperado de: http:// www.who.int/campaigns/world-health-day/2017/ es/

Piqueras, J., Ramos, V., Martínez, A., y Oblitas, L. (2009) Emociones negativas y su impacto en la salud mental y física. Suma Psicológica, 16(2), 85-112. Recuperado de http://www.redalyc.org/ pdf/1342/134213131007.pdf

Ramos-Brieva J, Cordero A. (1986). A new validation of the Hamilton Rating Scale for Depresión. Journal of psychiatric research, 22(1), 21-28.

Ríos, B., Rangel, G., Alvares, R., Castillo, F., Ramirez, G., Pantoja, J., Yañez, B., Arrieta, E. y Ruiz, K. (2008). Ansiedad, depresión y calidad de vida en el paciente obeso. Medigraphic Artemisa, 6(4).147-153. Recuperado de: http:// www.medigraphic.com/pdfs/actmed/am-2008/ am084a.pdf

Rudnicki, T. (2006). Aspectos emocionales del paciente renal crónico. Perspectivas en psicología, 2(2), 279 - 288. Recuperado: http:// www.redalyc.org/pdf/679/67920207.pdf Strohle, A. (2009). Physical activity, exercise, depression and anxiety disorders. En Journal of Neural Transmission, 116(6), 777-784. Recuperado de: https://www.ncbi.nlm.nih.gov/pubmed/18726137 
Tuesta, R. (2012). Calidad de vida relacionada con la salud. Elementos conceptuales. Salud. Revista Médica de Chile, 21(1), 71-76. Recuperado de: https://scielo.conicyt.cl/pdf/rmc/v138n3/art17.pdf

Villanueva, C., y Casas, J. (2014). Depresión y calidad de vida en pacientes con tratamiento de hemodiálisis en un Hospital de Es Salud en Lima - Perú, 2014. (Tesis para optar el título de Licenciado en Psicología). Universidad Peruana Unión.
Von Korff, M. y Simon, G. (1996) The relationship between pain and depression. The British Journal of Psychiatry. Supplement - Journals, 168 (30), 101-108. Recuperado: https://www.ncbi.nlm.nih. gov/pubmed/8864155

Recibido: 23/02/18

Aceptado: 21/04/18 